\title{
NMPC with Economic Objectives on Target Manifolds*
}

\author{
Niels van Duijkeren ${ }^{1}$, Timm Faulwasser ${ }^{2}$ and Goele Pipeleers ${ }^{1}$
}

\begin{abstract}
This paper presents a predictive control approach for stabilizing a target manifold in the state space of a nonlinear system while optimizing economic performance on/near this manifold. The control design is based on a transverse normal form description of the dynamics. While a stabilizing transversal NMPC acts as an outer control loop to stabilize a target neighborhood of the manifold, a tangential inner loop NMPC refines the remaining degrees of freedom to the benefit of economic performance without compromising manifold stability. The proposed two-stage approach is especially interesting for embedded system applications when the computationally attractive stabilizing transversal NMPC formulation is augmented with an "approximate" economic refinement step. We discuss the stability and performance properties of the resulting control scheme and show its efficacy in an illustrative example.
\end{abstract}

Index Terms - Nonlinear model predictive control, manifold stabilization, transverse normal forms, economic MPC

\section{INTRODUCTION}

In many control applications, the desired behavior is stated as a mix of objectives: certain outputs should converge to a reference, while other outputs ought to perform "optimally" according to some performance criteria. For instance, an autonomously driving car should remain close to the center of the lane while optimizing for driver comfort and fuel economy. In manufacturing applications, a robotic system should closely follow a prescribed geometric path while moving as fast as possible along it, in order to maximize productivity. In both cases a manifold is stabilized, while economic performance on the manifold should be optimized.

Transverse feedback linearization refers to the concept of finding, for a given an input-affine system and a controlledinvariant submanifold, a (locally) feedback equivalent system that is linear in the directions transverse to the manifold [1, 2]. Stabilization of the manifold can thereby be decoupled from controlling motion on the manifold. Path-following [39] is a special case where a one-dimensional output manifold is translated into a corresponding higher dimensional statespace manifold. Hence transverse normal forms (TNFs) are used for path-following controller synthesis; see e.g. [10]. In [8] TNFs are used to design a predictive path-following approach. The authors of [11] propose a two-stage NMPC

\footnotetext{
*NvD is fellow of the TEMPO Initial Training Network. The work is supported by the EU via ITN-TEMPO (607 957). The support from Flanders Make, the KU Leuven-BOF PFV/10/002 Centre of Excellence: Optimization in Engineering (OPTEC), and the Belgian Programme on Interuniversity Attraction Poles, initiated by the Belgian Federal Science Policy Office (DYSCO), is gratefully acknowledged. TF acknowledges funding from the Baden-Württemberg Stiftung under the Elite Programme for Postdocs.

${ }^{1}$ Department of Mechanical Engineering, KU Leuven, Belgium. \{niels.vanduijkeren, goele.pipeleers\}akuleuven.be

${ }^{2}$ Institute for Applied Computer Science, Karlsruhe Institute of Technology, Germany. timm. faulwasser@kit.edu
}

approach for manifold-stabilization using TNFs. Therein, it is proposed to solve a sequence of two OCPs at each time step, the first OCP optimizes the transverse part of the dynamics and the second OCP optimizes the tangential dynamics. We note that in [11], both OCPs have to be of tracking type, i.e. the cost function has to be lower bounded by a class $\mathcal{K}$ function.

Instead of tracking objectives, the by now large set of results on economic NMPC suggest to consider more general objectives [12]. In general, closed-loop stability, convergence and optimality are more difficult to address in this setting. Recent progress includes [13]: dissipativity-based methods employing a stabilizing terminal-constraint [14]; and approaches based on turnpike theory $[15,16]$. However, the progress on economic NMPC raises the question of how to trade off the dual objectives of economics and stability requirements. This is addressed in $[17,18]$ wherein a combination of economic and regulatory objectives is considered.

In the present paper, we discuss a two-stage approach for dual-objective NMPC applied to transverse feedback linearizable systems. We consider dual objectives where a target neighborhood of a controlled-invariant subset of a manifold is stabilized, while motion on the manifold shall be optimized according to a general performance objective. Similar to [11] we suggest solving two OCPs sequentially at every sampling instant. First an optimal control problem (OCP) is solved to stabilize the manifold. Secondly, the solution of the first OCP is used to establish convergence conditions of a neighborhood of the manifold. These conditions are then imposed in the second OCP that optimizes motion on the manifold. Extending the results of [11], we allow for explicit consideration of economic objectives on the target manifold. Furthermore, by enforcing convergence to the target neighborhood of the manifold, whose size is a design parameter, we allow for an explicit trade-off between economic performance and staying close to the target manifold. Quantification of the closed-loop economic NMPC performance with respect to the infinite horizon OCP [19] is difficult and beyond the scope of this paper. However, a numerical case-study illustrates that the closedloop performance is highly correlated to the size of the neighborhood.

The remainder of the paper is organized as follows. Section III briefly recapitulates transverse feedback linearization and presents the predictive control scheme that addresses the problem introduced in Section II. Section IV discusses recursive feasibility and convergence properties of the proposed control scheme. In Section V the results of a numerical case-study are discussed. Finally, Section VI draws the main 
conclusions of the paper.

\section{NOTATION}

The paper adopts the sampled-data setting for the presentation and analysis of the control scheme. At the sampling time $t_{i}$ the vector $\bullet\left(t_{i}\right)$ is written short as $\bullet_{i}$. Open-loop predictions of trajectories are denoted by $\bullet(\cdot)$, actual (closedloop) trajectories are denoted as $\bullet(\cdot)$. Optimal open-loop trajectories are prepended with a super-script, e.g., $\pitchfork \cdot(\cdot)$ is optimal to $\mathrm{OCP}^{\pitchfork}$ (to be introduced).

\section{PROBLEM STATEMENT}

Consider the given nonlinear input-affine system

$$
\begin{aligned}
& \dot{x}=f(x)+\sum_{i=1}^{m} g_{i}(x) u_{i}=: f(x)+G(x) u \\
& y=h(x),
\end{aligned}
$$

with state $x \in \mathbb{R}^{n}$, input $u \in \mathcal{U} \subset \mathbb{R}^{m}$, and outputs $y \in \mathbb{R}^{p}$. The vector fields $f, g_{1}, \ldots, g_{m}: \mathbb{R}^{n} \rightarrow \mathbb{R}^{n}$ are smooth and we assume $g_{1}, \ldots, g_{m}$ are linearly independent. We consider a manifold in the state space given by

$$
\mathcal{M}=\left\{x \in \mathbb{R}^{n} \mid \sigma(h(x))=0\right\},
$$

with $\sigma: \mathbb{R}^{p} \rightarrow \mathbb{R}^{l}, l \leq m$. Since the manifold $\mathcal{M}$ is generally not controlled invariant, we denote $\mathcal{M}^{\star}$ to be the maximum controlled invariant subset contained in $\mathcal{M}$, controlled by $u \in \mathcal{U}$.

Assumption 1 (Feasibility of manifold stabilization): The maximal controlled invariant subset of $\mathcal{M}$, denoted by $\mathcal{M}^{\star} \subseteq \mathbb{R}^{n}$, is a non-empty, closed embedded submanifold of the state space with dimension $n^{\star}$.

Assumption 1 is a basic feasibility requirement for the manifold stabilization problem, meaning that if $x_{0} \in \mathcal{M}^{\star}$ there exist controls $u(t) \in \mathcal{U}$ such that $x(t) \in \mathcal{M}^{\star}, \forall t \geq t_{0}$.

We aim to find an efficient method to approximately solve OCPs of the following nature:

$$
\begin{aligned}
\min _{\bar{x}(\cdot), \bar{u}(\cdot)} & J_{\text {eco }}(\bar{x}(\cdot), \bar{u}(\cdot)) \\
\text { s.t. } & \overline{\bar{x}}(\tau)=f(\bar{x}(\tau))+G(\bar{x}(\tau)) \bar{u}(\tau) \quad \forall \tau \\
& \bar{x}\left(t_{i}\right)=x_{i} \\
& \lim _{\tau \rightarrow \infty} \operatorname{dist}\left(\mathcal{M}^{\star}, \bar{x}(\tau)\right) \leq \epsilon \\
& \bar{u}(\tau) \in \mathcal{U} \quad \forall \tau,
\end{aligned}
$$

with $u(\cdot) \in \mathcal{L}^{\infty}([0, \infty), \mathcal{U})$ and $J_{\text {eco }}: \mathcal{L}^{\infty}\left(\mathbb{R}, \mathbb{R}^{n}\right) \times$ $\mathcal{L}^{\infty}(\mathbb{R}, \mathcal{U}) \rightarrow \mathbb{R}$ an economic performance objective on, respectively, close to $\mathcal{M}^{\star}$. The above problem is generally complex and usually tackled by an offline optimization phase, accompanied by an online reference tracking phase.

\section{PROPOSED SOLUTION}

We propose tackling OCP (3) by dividing it into two sequential subproblems: A first OCP designed to stabilize an $\mathcal{N}$-neighborhood of the manifold, and a second OCP achieving the desired economic motion tangential to the manifold. Both of which are to be solved at every sampling instance. First we introduce the transverse normal form (TNF) for the input-affine system (1).

\section{A. TRANSVERSE NORMAL FORMS}

Assumption 2 (Vector relative degree): There exist functions $\alpha_{1}(x), \ldots, \alpha_{l}(x): \mathbb{R}^{n} \rightarrow \mathbb{R}$ such that

(a) $\mathcal{M}^{\star} \subset\left\{x \in \mathbb{R}^{n} \mid \alpha_{1}(x)=\ldots=\alpha_{l}(x)=0\right\}$, and

(b) $\alpha(x):=\left[\alpha_{1}(x) \cdots \alpha_{l}(x)\right]^{\top}$ yields a well-defined vector relative degree $\rho=\left\{\rho_{1}, \ldots, \rho_{l}\right\}$ on $\mathbb{R}^{n}$ with $\sum_{i=1}^{l} \rho_{i}=$ $n-n^{\star}$, cf. [20, 21].

The authors of [1] show that if Assumptions 1 and 2 hold, then locally around any point of $\mathcal{M}^{\star}$ there exists a state transformation $\Phi: \mathbb{R}^{n} \rightarrow \mathbb{R}^{n-n^{\star}} \times \mathbb{R}^{n^{\star}}, x \mapsto(\xi, \eta)$, and a transversely linearizing feedback

$$
u=a(\xi, \eta)+b(\xi, \eta)\left[v^{\pitchfork^{\top}} v^{\| \mathbf{\top}}\right]^{\top}=\kappa\left(\xi, \eta, v^{\pitchfork}, v^{\|}\right),
$$

such that in the $(\xi, \eta)$-coordinates system (1) reads

$$
\begin{aligned}
& \dot{\xi}=A \xi+B v^{\pitchfork} \\
& \dot{\eta}=f^{0}(\xi, \eta)+G^{\pitchfork}(\xi, \eta) v^{\pitchfork}+G^{\|}(\xi, \eta) v^{\|},
\end{aligned}
$$

with the pair $(A, B)$ controllable and the image $\Phi\left(\mathcal{M}^{\star}\right)=$ $\{(\xi, \eta) \mid \xi=0\}$. The transverse dynamics are described by differential equations (5a), which resemble a set of $l$ integrator chains, with $\xi=\left[\alpha_{1} \cdots \alpha_{1}^{\left(\rho_{1}-1\right)} \cdots \alpha_{l} \cdots \alpha_{l}^{\left(\rho_{l}-1\right)}\right]^{\top} \in$ $\mathbb{R}^{n-n^{\star}}$ and $v^{\pitchfork}=\left[\alpha_{1}^{\left(\rho_{1}\right)} \cdots \alpha_{l_{\star}}^{\left(\rho_{l}\right)}\right]^{\top} \in \mathbb{R}^{l}$. The tangential dynamics, with state $\eta \in \mathbb{R}^{n^{\star}}$ and the tangential input $v^{\|} \in \mathbb{R}^{m-l}$ are governed by (5b). This set of, generally nonlinear, differential equations describes the motion on $\mathcal{M}^{\star}$. The virtual output function is chosen as $\alpha=\sigma \circ h$ and verified for Assumption 2.

Remark 1: Note that more general classes of systems can be considered, such as quasi-static transverse feedback linearizable systems with manifolds in flat output spaces, see $[11,22]$.

Remark 2: Since $\Phi$ is a diffeomorphism, the relation between $(\xi, \eta)$ and $x$ is bijective and invertible. To denote the dependency of a function on $\xi, \eta$ or the pair $(\xi, \eta)$, we often write $x$ instead. Furthermore, let $v(\cdot)$ be short-hand notation for both the vector concatenation $\left[v^{\pitchfork}(\cdot)^{\top} v^{\|}(\cdot)^{\top}\right]^{\top}$, and the pair $\left(v^{\pitchfork}(\cdot), v^{\|}(\cdot)\right)$.

\section{B. PROPOSED SEQUENTIAL DUAL-OBJECTIVE NMPC}

Now let us regard the NMPC control scheme solving two OCPs sequentially in a receding horizon fashion.

The time horizon at time $t_{i}$ is defined by $\Omega_{i}=\left[t_{i}, t_{i}+T\right]$. Consider a tracking-type objective for the manifold stabilization loop

$$
\begin{aligned}
& J^{\pitchfork}\left(\bar{\xi}(\cdot), \bar{v}^{\pitchfork}(\cdot)\right)= \\
& \quad \int_{\Omega_{i}} F^{\pitchfork}\left(\bar{\xi}(\tau), \bar{v}^{\pitchfork}(\tau)\right) \mathrm{d} \tau+E\left(\bar{\xi}\left(t_{i}+T\right)\right),
\end{aligned}
$$

Assumption 3 (Positive-definite cost function): The Lagrange cost $F^{\pitchfork}: \mathbb{R}^{n-n^{\star}} \times \mathbb{R}^{l} \rightarrow \mathbb{R}$ is specified by positive definite matrices $Q, R \succ 0$ such that

$$
F^{\pitchfork}\left(\xi, v^{\pitchfork}\right)=\|\xi\|_{Q}^{2}+\left\|v^{\pitchfork}\right\|_{R}^{2} \geq \gamma(\|\xi\|), \quad \forall \xi, v^{\pitchfork},
$$

where $\gamma$ is of class $\mathcal{K}$ and the terminal penalty $E(\xi)=\|\xi\|_{P}^{2}$, with $P \succ 0$ the solution of the algebraic Riccati equation

$$
A^{\top} P+P A+Q=P B R^{-1} B^{\top} P \text {. }
$$


The cost functional according to which the tangential trajectories are optimized is

$$
J^{\|}\left(\bar{\xi}(\cdot), \bar{\eta}(\cdot), \bar{v}^{\pitchfork}(\cdot), \bar{v}^{\|}(\cdot)\right)=\int_{\Omega_{i}} F^{\|}(\bar{\xi}(\tau), \bar{\eta}(\tau), \bar{v}(\tau)) \mathrm{d} \tau .
$$

Note that $J^{\|}$is chosen in a way such that, close to $\mathcal{M}^{\star}$, it approximates $J_{\text {eco }}$. Futhermore, we denote the terminal constraint sets by $\mathcal{E}_{\xi}, \mathcal{E}_{\eta}$ and define the neighborhood $\mathcal{N}=$ $\left\{\xi \in \mathbb{R}^{n-n^{\star}} \mid E(\xi) \leq \epsilon_{\mathcal{N}}^{2}\right\} \subseteq \mathcal{E}_{\xi}$, a level-set of the terminal cost in (6).

Algorithm 1: Given a sampling time interval $\delta>0$, for sampling time instants $t_{i}=i \delta, i \in \mathbb{N}_{0}$, the control NMPC feedback law is determined by the algorithm below:

1) Measure or estimate initial state $x_{i} \in \mathbb{R}^{n}$.

2) Solve $\operatorname{OCP}^{\pitchfork}\left(x_{i}\right)$

$$
\begin{array}{ll}
\min & J^{\pitchfork}\left(\bar{\xi}(\cdot), \bar{v}^{\pitchfork}(\cdot)\right) \\
\text { s.t. } & \dot{\bar{\xi}}=A \bar{\xi}+B \bar{v}^{\pitchfork} \\
& \dot{\bar{\eta}}=f^{0}(\bar{\xi}, \bar{\eta})+G^{\pitchfork}(\bar{\xi}, \bar{\eta}) \bar{v}^{\pitchfork}+G^{\|}(\bar{\xi}, \bar{\eta}) \bar{v}^{\|} \\
& {\left[\bar{\xi}_{i}^{\top} \bar{\eta}_{i}^{\top}\right]^{\top}=\Phi\left(x_{i}\right)} \\
& \kappa\left(\bar{\xi}, \bar{\eta}, \bar{v}^{\pitchfork}, \bar{v}^{\|}\right) \in \mathcal{U} \\
& \bar{\xi}\left(t_{i}+T\right) \in \mathcal{E}_{\xi} \quad \bar{\eta}\left(t_{i}+T\right) \in \mathcal{E}_{\eta},
\end{array}
$$

and denote the optimal solution trajectories by $\pitchfork \bar{\xi}\left(\cdot ; x_{i}\right)$, ${ }^{\pitchfork} \bar{\eta}\left(\cdot ; x_{i}\right),{ }^{\pitchfork} \bar{v}^{\pitchfork}\left(\cdot ; x_{i}\right),{ }^{\pitchfork} \bar{v}^{\|}\left(\cdot ; x_{i}\right)$.

3) Solve $\operatorname{OCP}^{\|}\left(x_{i}\right)$, optimizing economic performance:

$$
\begin{array}{ll}
\min & J^{\|}\left(\bar{\xi}(\cdot), \bar{\eta}(\cdot), \bar{v}^{\pitchfork}(\cdot), \bar{v}^{\|}(\cdot)\right) \\
\text { s.t. } & \dot{\bar{\xi}}=A \bar{\xi}+B \bar{v}^{\pitchfork} \\
& \dot{\bar{\eta}}=f^{0}(\bar{\xi}, \bar{\eta})+G^{\pitchfork}(\bar{\xi}, \bar{\eta}) \bar{v}^{\pitchfork}+G^{\|}(\bar{\xi}, \bar{\eta}) \bar{v}^{\|} \\
& {\left[\bar{\xi}_{i}^{\top} \bar{\eta}_{i}^{\top}\right]^{\top}=\Phi\left(x_{i}\right)} \\
& \kappa\left(\bar{\xi}, \bar{\eta}, \bar{v}^{\pitchfork}, \bar{v}^{\|}\right) \in \mathcal{U} \\
& E(\bar{\xi}(\tau)) \leq \max \left\{\epsilon_{\mathcal{N}}^{2}, E\left(^{\pitchfork} \bar{\xi}\left(\tau ; x_{i}\right)\right)\right\} \forall \tau \in \Omega_{i}
\end{array}
$$

and enforcing the convergence/invariance conditions:

$$
\begin{array}{ll}
\text { s.t. } & \text { if } J^{\pitchfork}\left({ }^{\pitchfork} \bar{\xi}\left(\cdot ; x_{i}\right),{ }^{\pitchfork} \bar{v}^{\pitchfork}\left(\cdot ; x_{i}\right)\right)=E\left(\xi_{i}\right) \leq \epsilon_{\mathcal{N}}^{2} \text { : } \\
& \dot{\hat{\xi}}=(A-B K) \hat{\xi}, \hat{\xi}_{i+1}=\bar{\xi}_{i+1}, \hat{v}^{\pitchfork}=-K \hat{\xi} \\
& \text { else } \hat{\xi}={ }^{\pitchfork} \bar{\xi}, \hat{v}^{\pitchfork}={ }^{\pitchfork} \bar{v}^{\pitchfork}, \bar{\xi}_{i+1}={ }^{\pitchfork} \bar{\xi}_{i+1} \text { end } \\
& \dot{\hat{\eta}}=f^{0}(\hat{\xi}, \hat{\eta})+G^{\pitchfork}(\hat{\xi}, \hat{\eta}) \hat{v}^{\pitchfork}+G^{\|}(\hat{\xi}, \hat{\eta}) \hat{v}^{\|} \\
& \hat{\eta}_{i+1}=\bar{\eta}_{i+1} \\
& \kappa\left(\hat{\xi}, \hat{\eta}, \hat{v}^{\pitchfork}, \hat{v}^{\|}\right) \in \mathcal{U} \\
& \hat{\eta}\left(t_{i}+T\right) \in \mathcal{E}_{\eta},
\end{array}
$$

with $K=R^{-1} B^{\top} P$. Denote the optimal solution trajectories by ${ }^{\|} \bar{\xi}\left(\cdot ; x_{i}\right),{ }^{\|} \bar{\eta}\left(\cdot ; x_{i}\right),{ }^{\|} \bar{v}^{\pitchfork}\left(\cdot ; x_{i}\right),{ }^{\|} \bar{v}^{\|}\left(\cdot ; x_{i}\right)$.

4) Using the optimal trajectories from $\mathrm{OCP}^{\|}$, set

$$
{ }^{\|} \bar{u}\left(\cdot ; x_{i}\right)=\kappa\left({ }^{\|} \bar{\xi}\left(\cdot ; x_{i}\right),{ }^{\|} \bar{\eta}\left(\cdot ; x_{i}\right),{ }^{\|} \bar{v}^{\pitchfork}\left(\cdot ; x_{i}\right),{ }^{\|} \bar{v}^{\|}\left(\cdot ; x_{i}\right)\right) .
$$

5) Apply $u_{\mathrm{MPC}}(t)=\|_{\bar{u}}\left(t ; x_{i}\right), t_{i} \leq t<t_{i}+\delta$ and at time instance $t_{i}+\delta$ repeat the procedure from 1 ).

$\mathrm{OCP}^{\dagger}$ is designed such that nominal convergence of $\xi$ to the origin is achieved. This is for instance the case when $Q$,
$R$ and $P$ are chosen according to Assumption 3 and $\mathcal{E}_{\xi}$ and $\mathcal{E}_{\eta}$ are suitable terminal constraint sets. The second OCP\| optimizes the motion tangent to the manifold. Convergence to the target neighborhood $\mathcal{N}$ is enforced using $(10 \mathrm{~g})-(10 \mathrm{~m})$. $\mathrm{OCP}^{\|}$explicitly plans an additional converging $\hat{\xi}$-trajectory from the predicted state at the next sampling time instance. If $\xi_{i}$ is outside the target neighborhood, the planned $\bar{\xi}$-trajectory from $\mathrm{OCP}^{\pitchfork}$ should remain feasible. If $\xi_{i}$ is inside the target neighborhood and the LQR feedback is recursively feasible, a closed-loop LQR trajectory must also be feasible from the next sampling time instance. The sequential structure of the algorithm explicitly prioritizes stabilization of the set $\mathcal{N}$ over the economic objective. Choosing an aggressive tuning in $\mathrm{OCP}^{\pitchfork}(Q \gg R)$ improves the convergence rate at the cost of economic performance outside the set $\mathcal{N}$.

\section{IMPLEMENTATION}

We point out that the structure of the TNF admits numerically attractive properties. The numerical implementation of the OCPs in Algorithm 1 can be approached by approximating them by nonlinear programs (NLPs) using a direct multiple-shooting method. The NLP approximation of (9) forms a constrained least-squares problem and can therefore be efficiently solved using the Generalized Gauss-Newton method.

In general, the NLP approximation of (10) is much more challenging to solve efficiently. However, since $\mathrm{OCP}^{\pitchfork}$ already generates a suboptimal solution to $\mathrm{OCP}^{\|}$, any improvement that can be made in the given time will be in the benefit of closed-loop optimality without the need to compromise convergence to $\mathcal{N}$. We argue that it suffices to use an approximate solution strategy for which optimality is not necessarily attained at each sampling instance, but rather as time progresses. This is a well-known concept used in real-time iteration type optimization schemes [23-25].

\section{PROPERTIES OF CONTROL SCHEME}

Next we establish formal properties of the proposed control scheme. Throughout the analysis, statements of the form: "The closed-loop system defined by (9) and (5a)" will refer to the system (5a) being controlled via an NMPC control law according to OCP (9) as defined below.

Definition 1 (Sampled-data NMPC control law): For $t \in$ $\left[t_{i}, t_{i}+\delta\right)$, the inputs are the solutions $\cdot \bar{v}^{\pitchfork}\left(t ; x_{i}\right)$ and $\bar{v}^{\|}\left(t ; x_{i}\right)$ of $\mathrm{OCP}^{\cdot}\left(x_{i}\right)$, with $\bullet \in\{\pitchfork, \|\}$.

Assumption 4 (Continuity of system dynamics): The vector fields $f, g_{1}, \ldots, g_{m}: \mathbb{R}^{n} \rightarrow \mathbb{R}^{n}$ are sufficiently often continuously differentiable.

Assumption 5 (Continuity of system trajectories): For any $x_{0} \in \mathbb{R}^{n}$ and any input function $u(\cdot) \in \mathcal{L}^{\infty}(\mathbb{R}, \mathcal{U})$ the system (1) has an absolutely continuous solution.

We assume the existence of open-loop inputs in the terminal set, and define the notions of an admissible set and a maximum admissible set.

Assumption 6 (Existence of terminal controls): For all $(\tilde{\xi}, \tilde{\eta}) \in \mathcal{E}_{\xi} \times \mathcal{E}_{\eta}$ there exist input signals $v_{\mathcal{E}_{\xi}}^{\pitchfork}(\cdot) \in \mathcal{L}^{\infty}$ and 


$$
\begin{aligned}
& v_{\mathcal{E}_{\eta}}^{\|}(\cdot) \in \mathcal{L}^{\infty} \text {, such that } \forall \tau \in[0, \delta] \\
& \kappa\left(\xi\left(\tau ; \tilde{x}, v_{\mathcal{E}_{\xi}}^{\pitchfork}(\cdot)\right), \eta\left(\tau ; \tilde{x}, v_{\mathcal{E}}^{\pitchfork}(\cdot)\right), v_{\mathcal{E}_{\xi}}^{\pitchfork}(\tau), v_{\mathcal{E}_{\eta}}^{\pitchfork}(\tau)\right) \in \mathcal{U}, \\
& \xi\left(\tau ; \tilde{x}, v_{\mathcal{E}_{\xi}}^{\pitchfork}(\cdot)\right) \in \mathcal{E}_{\xi}, \\
& \eta\left(\tau ; \tilde{x}, v_{\mathcal{E}}(\cdot)\right) \in \mathcal{E}_{\eta},
\end{aligned}
$$

with $v_{\mathcal{E}}=\left[\begin{array}{ll}v_{\mathcal{E}_{\xi}}^{\pitchfork \boldsymbol{\top}} & v_{\mathcal{E}_{\eta}}^{\| \boldsymbol{\top}}\end{array}\right]^{\top}$, and

$$
\begin{aligned}
& E\left(\xi\left(\tau ; \tilde{x}, v_{\mathcal{E}_{\xi}}^{\pitchfork}(\cdot)\right)\right)-E(\tilde{\xi}) \leq \\
& -\int_{0}^{\tau} F^{\pitchfork}\left(\xi\left(s ; \tilde{x}, v_{\mathcal{E}_{\xi}}^{\pitchfork}(\cdot)\right), v_{\mathcal{E}_{\xi}}^{\pitchfork}(s)\right) \mathrm{d} s, \forall \tau \in[0, \delta] .
\end{aligned}
$$

Definition 2 (Admissible state set $X$ ): A set $X \subseteq \mathbb{R}^{n}$ is called admissible if for all $x_{0} \in X$ there exists an admissible input $\tilde{v}(\cdot) \in \mathcal{L}^{\infty}$, such that

1) $\kappa\left(\xi\left(\tau ; x_{0}, \tilde{v}(\cdot)\right), \eta\left(\tau ; x_{0}, \tilde{v}(\cdot)\right), \tilde{v}(\cdot)\right) \in \mathcal{U}, \tau \in[0, T]$,

2) $\xi\left(T ; x_{0}, \tilde{v}(\cdot)\right) \in \mathcal{E}_{\xi}$,

3) $\eta\left(T ; x_{0}, \tilde{v}(\cdot)\right) \in \mathcal{E}_{\eta}$.

Definition 3 (Maximum admissible set $\mathcal{R}$ ): The set $\mathcal{R}$ denotes the maximum admissible set, such that (9) admits, for all $x_{0} \in \mathcal{R}$, an optimal (not necessarily unique) solution.

Definition 4 (Distance to a set): The distance of a point $\xi$ to a set $\mathcal{N}$ is denoted as $\|\xi\|_{\mathcal{N}}=\min _{z \in \mathcal{N}}\|\xi-z\|_{2}$.

Proposition 1 (Recursive feasibility): Let Assumption 6 hold. If $\mathrm{OCP}^{\dagger}$ is feasible at time $t=t_{0}$, then the sequence of $\mathrm{OCP}^{\dagger}$ and $\mathrm{OCP}^{\|}$, in closed-loop with (5a) is feasible for all $t_{i} \geq t_{0}$.

Proof: Consider any recalculation instant $t_{i}$, for which a solution exists to $\mathrm{OCP}^{\dagger}$ (e.g., $t_{0}$ ). The optimal solution of $\mathrm{OCP}^{\mathrm{h}}$ satisfies (10b)-(10f). To show that these trajectories also satisfy constraints $(10 \mathrm{~g})-(10 \mathrm{~m})$, the two possible branches of the if-statement are considered. Consider $J^{\dagger}\left({ }^{\dagger} \bar{\xi}\left(\cdot ; x_{i}\right),{ }^{\dagger} \bar{v}^{\dagger}\left(\cdot ; x_{i}\right)\right)=E\left(\xi_{i}\right) \leq \epsilon_{\mathcal{N}}^{2}$, this implies $\xi\left(t_{i}\right) \in \mathcal{N} \subseteq \mathcal{E}_{\xi}$ and that the transverse cost functional (6) evaluates to the unconstrained infinite-horizon LQR cost. Given the uniqueness of the LQR solution, the optimal solution of $\mathrm{OCP}^{\dagger}$ coincides with the infinite horizon LQR trajectory and is therefore a feasible candidate to (10h). For both branches of $(10 \mathrm{~g})$, the optimal solution of $\mathrm{OCP}^{\mathrm{t}}$ satisfies (10c)- $(10 \mathrm{~m})$. Hence feasibility of $\mathrm{OCP}^{\dagger}$ implies feasibility of OCP ${ }^{\|}$. Due to constraints $(10 \mathrm{~g})-(10 \mathrm{~m})$ a trajectory exist from $\left(\| \bar{\xi}\left(t_{i+1} ; x_{i}\right),{ }^{\|} \bar{\eta}\left(t_{i+1} ; x_{i}\right)\right)$ which can, by Assumption 6, be extended for a time interval $\delta$. Since there are no disturbances and no plant-model mismatch, $\xi(\tau)=\| \bar{\xi}\left(\tau ; x_{i}\right), \eta(\tau)={ } \bar{\eta}\left(\tau ; x_{i}\right), \tau \in\left[t_{i}, t_{i+1}\right]$. Picking any such input we obtain as an admissible input for $t=t_{i+1}$

$$
\tilde{v}\left(\tau ; x_{i+1}\right)=\left\{\begin{array}{l}
\| \hat{v}^{\dagger}\left(\tau ; x_{i}\right), \tau \in\left[\delta, T_{i}\right] \\
v_{\mathcal{E}}\left(\tau-T_{i}\right), \tau \in\left(T_{i}, T_{i}+\delta\right]
\end{array},\right.
$$

where $T_{i}$ is short for $t_{i}+T$. Hence, feasibility of OCP $\|$ at $t_{i}$ implies feasibility of $\mathrm{OCP}^{\mathrm{\dagger}}$ at $t_{i+1}$.

Proposition 2 (Convergence of $\xi(\cdot)$ to $\mathcal{N})$ : Suppose that Assumptions 3-6 hold. Then the system defined by the sequence $\mathrm{OCP}^{\mathrm{H}}$ and $\mathrm{OCP}^{\|}$, in closed loop with (5a) achieves $\lim _{t \rightarrow \infty}\|\xi(t)\|_{\mathcal{N}}=0 \forall x_{0} \in \mathcal{R}$.

Proof: Step 1: First we show that for (5a) in closed-loop with $\mathrm{OCP}^{\pitchfork}\|\xi(t)\| \rightarrow 0, t \rightarrow \infty$. This part of the proof is analogous to well-known results by e.g., [26, 27]. Consider the optimal value function $V\left(x_{i}\right)$ resulting from evaluating $J^{\pitchfork}$ with the optimal trajectories $\pitchfork \bar{\xi}\left(\cdot ; x_{i}\right)$ and ${ }^{\pitchfork} \bar{v}^{\pitchfork}\left(\cdot ; x_{i}\right)$ from $\mathrm{OCP}^{\pitchfork}$ and the cost $J^{\pitchfork}\left(\bar{\xi}\left(\cdot ; x_{i+1}, \tilde{v}\left(\cdot ; x_{i+1}\right)\right), \tilde{v}\left(\cdot ; x_{i+1}\right)\right)$ starting from $x_{i+1}$ using input $\tilde{v}\left(\cdot ; x_{i+1}\right)$ as defined in (13). Subtracting the two yields

$$
\begin{aligned}
& J^{\dagger}\left(\bar{\xi}\left(\cdot ; x_{i+1}, \tilde{v}\left(\cdot ; x_{i+1}\right)\right), \tilde{v}\left(\cdot ; x_{i+1}\right)\right)-V\left(x_{i}\right)= \\
& -\int_{t_{i}}^{t_{i}+\delta} F^{\dagger}\left({ }^{\dagger} \bar{\xi}\left(\tau ; x_{i}\right),{ }^{\dagger} \bar{v}^{\dagger}\left(\tau ; x_{i}\right)\right) \mathrm{d} \tau \\
& +\int_{T_{i}}^{T_{i}+\delta} F^{\pitchfork}\left(\bar{\xi}\left(\tau ; x_{i+1}, \tilde{v}\left(\cdot ; x_{i+1}\right)\right), \tilde{v}\left(\tau ; x_{i+1}\right)\right) \mathrm{d} \tau \\
& -E\left({ }^{\pitchfork} \bar{\xi}\left(T_{i} ; x_{i}\right)\right)+E\left(\bar{\xi}\left(T_{i}+\delta ; x_{i+1}, \tilde{v}\left(\cdot ; x_{i+1}\right)\right)\right) .
\end{aligned}
$$

By evaluating (12) for the interval $\left[T_{i}, T_{i}+\delta\right]$, starting from ${ }^{\dagger} \xi\left(T_{i} ; x_{i}\right)$ we obtain zero as an upper bound for the last three terms in the expression above. Furthermore, because $V\left(x_{i+1}\right) \leq J^{\dagger}\left(\bar{\xi}\left(\cdot ; x_{i+1}, \tilde{v}\left(\cdot ; x_{i+1}\right)\right), \tilde{v}\left(\cdot ; x_{i+1}\right)\right)$ and $F^{\dagger}$ is lower bounded by $\gamma \in \mathcal{K}$, the next inequality follows:

$$
V\left(x_{i+1}\right)-V\left(x_{i}\right) \leq-\int_{t_{i}}^{t_{i}+\delta} \gamma\left({ }^{\dagger} \bar{\xi}\left(\tau ; x_{i}\right)\right) \mathrm{d} \tau .
$$

The value function strictly decreases in between $t_{i}$ and $t_{i+1}$. Also,

$$
\begin{aligned}
& V\left(x_{N}\right)-V\left(x_{0}\right)=\sum_{i=0}^{N-1} V\left(x_{i+1}\right)-V\left(x_{i}\right) \\
& \leq-\int_{t_{0}}^{t_{N}} \gamma\left(\xi\left(\tau ; x_{0},{ }^{\dagger} v^{\dagger}\left(\cdot ; x_{0}\right)\right)\right) \mathrm{d} \tau,
\end{aligned}
$$

where ${ }^{\pitchfork} v^{\pitchfork}\left(\cdot ; x_{0}\right)$ denotes the closed-loop input trajectory according to the NMPC control law OCP ${ }^{\pitchfork}$. Since by Assumption $3 V(\tilde{\xi}) \geq 0 \forall \tilde{\xi}$, we deduce that for $t_{N} \rightarrow \infty$ the right-hand-side of the inequality is bounded. Moreover, due to Assumptions 4 and 5 and Definition 3 it follows that $\xi(\cdot), \dot{\xi}(\cdot) \in \mathcal{L}^{\infty}$, by Barbalat's Lemma [28, Lem. 4] we can then conclude that $\lim _{t_{N} \rightarrow \infty}\left\|\xi\left(t_{N} ; x_{0},{ }^{\dagger} v^{\pitchfork}\left(\cdot ; x_{0}\right)\right)\right\|=0$ $\forall x_{0} \in \mathcal{R}$.

Step 2: Secondly, we show that the NMPC control law obtained by solving (9) and (10) sequentially, applied to system $(5 \mathrm{a}), \lim _{t \rightarrow \infty}\|\xi(t)\|_{\mathcal{N}}=0$. Consider any recalculation instant $t_{i}$ when the if-condition $(10 \mathrm{~g})$ is false. Since the origin is in the interior of $\mathcal{N}$, constraint (10i) enforces the $\xi$-trajectory to follow ${ }^{\pitchfork} \xi\left(\cdot ; x_{i}\right)$ which is shown to converge to the origin, and therefore to the set $\mathcal{N}$. Now, consider a recalculation instant $t_{i}$ when $J^{\dagger}\left({ }^{\dagger} \bar{\xi}\left(\cdot ; x_{i}\right),{ }^{\dagger} \bar{v}^{\pitchfork}\left(\cdot ; x_{i}\right)\right)=$ $E\left(\xi_{i}\right) \leq \epsilon_{\mathcal{N}}^{2}$. The infinite horizon LQR control law renders any sublevel set of the according Lyapunov function $\xi \mapsto$ $\xi^{\top} P \xi$ forward invariant. Moreover, it is well-known that under Assumption 3, the cost functional (6) in $\mathrm{OCP}^{\mathrm{t}}$ is lower-bounded by the LQR cost and this lower bound is attained when feasible. Hence, (10h) enforces the optimal trajectories from $\mathrm{OCP}^{\dagger}$ to be forward invariant with respect to $\mathcal{N} \subseteq \mathcal{E}_{\xi}$, a sublevel set of the LQR Lyapunov function. Constraint (10f) thereby enforces the state $\xi(t)$ to remain inside the set $\mathcal{N}, \forall t \geq t_{i}$. 
Theorem 1: Suppose that Assumptions 1-6 hold. Then the closed-loop system defined by Algorithm 1 and (1) admits the following properties for all $x_{0} \in \mathcal{R}$ :

(a) Asymptotic convergence to an $\epsilon$-neighborhood of the manifold $\mathcal{M}^{\star}$, i.e. $\lim _{t \rightarrow \infty}\|\alpha(x(t))\|_{2} \leq \epsilon$.

(b) The input constraints $u \in \mathcal{U}$ are satisfied.

Proof: Consider the vector $\xi_{1}$ corresponding to $\alpha_{1}, \ldots, \alpha_{l}$ and $\xi_{2}$ the vector of remaining elements corresponding to the first and higher time derivatives of $\alpha_{1}, \ldots, \alpha_{l}$. In Proposition 2 it is established that $\lim _{t \rightarrow \infty}\|\xi(t)\|_{P} \leq \epsilon_{\mathcal{N}}$. Let us consider the set $\mathcal{N}_{2}=\left\{\xi_{1} \in\right.$ $\left.\mathbb{R}^{l} \mid \exists \xi_{2}:\|\xi(t)\|_{P} \leq \epsilon_{\mathcal{N}}\right\}$. For $\mathcal{B}(r)=\left\{\xi_{1} \in \mathbb{R}^{l} \mid\left\|\xi_{1}\right\|_{2} \leq\right.$ $r\}$, we characterize $\epsilon=\min \left\{r \in \mathbb{R} \mid \mathcal{N}_{1} \subseteq \mathcal{B}(r)\right\}$. The value of $\epsilon$ exists and is finite, since the set $\mathcal{N}$ is compact. Assumptions 1 and 2 are sufficient for (1) to be equivalent to (5) under the transversely linearizing feedback (4) [1, Thm. 3.2]. Convergence of $\xi$ to a neighborhood $\mathcal{N}$ of the origin is equivalent to convergence to an $\epsilon$-distance of the manifold $\mathcal{M}^{\star}$ in the state-space of (1), which concludes $(a)$. The NMPC scheme described in Algortihm 1 explicitly handles the input constraints $u \in \mathcal{U}$ using the input transformation $\kappa$. Recursive feasibility was shown in Proposition 1 and thereby we conclude item $(b)$ holds true.

\section{SIMULATION EXAMPLE}

The simulation study considers a model predictive pathfollowing control (MPFC) problem applied to a planar robotic manipulator, see [8] for more details regarding MPFC. The closed-loop economic performance is evaluated for different values of $\epsilon_{\mathcal{N}}$. The implementation of the example uses CasADi [29] to model the OCP, algorithmic differentiation and the interface to the interior-point NLP solver IPOPT [30].

\section{A. 2-DOF ROBOT MANIPULATOR}

Consider the state space description of a fully-actuated rigid-body 2-DOF serial link manipulator with the state vector $x$, augmented with virtual states $z$ describing the evolution of the path parameter under virtual input $w \in \mathbb{R}$ :

$$
\begin{aligned}
& \dot{x}=\left[\begin{array}{c}
x_{2} \\
B^{-1}\left(x_{1}\right)\left(u-C\left(x_{1}, x_{2}\right) x_{2}-g\left(x_{1}\right)-F_{v} x_{2}\right)
\end{array}\right] \\
& \dot{z}=\left[\begin{array}{ll}
0 & 1 \\
0 & 0
\end{array}\right] z+\left[\begin{array}{l}
0 \\
1
\end{array}\right] w \\
& y=h(x):=x_{1}-p(\theta) \\
& \theta=z_{1},
\end{aligned}
$$

where $x_{1} \in \mathbb{R}^{2}$ and $x_{2} \in \mathbb{R}^{2}$ denote the vector of joint angles and joint velocities, respectively. Furthermore, $u=$ $\left[\begin{array}{ll}u_{1} & u_{2}\end{array}\right]^{\top} \in \mathbb{R}^{2}$ denotes the vector of joint torques.

If $\sigma$ is the identity, $\alpha(x)=\sigma(h(x))$ satisfies Assumption 2 with a vector relative degree of $\left\{\rho_{1}, \rho_{2}\right\}=\{2,2\}$. For this system, a transversely linearizing feedback is

$$
\begin{aligned}
& u=B\left(x_{1}\right)\left(v^{\pitchfork}+\frac{\partial^{2} p(\theta)}{\partial \theta^{2}} \dot{\theta}^{2}+\frac{\partial p(\theta)}{\partial \theta} v^{\|}\right)+ \\
& +C\left(x_{1}, x_{2}\right) x_{2}+g\left(x_{1}\right)+F_{v} x_{2}, \\
& w=v^{\|} \text {. }
\end{aligned}
$$

TABLE I

PROPERTIES OF THE SIMULATED PLANAR REVOLUTE JOINT ROBOT ARM.

\begin{tabular}{|c|c|c|}
\hline Property & Link/Joint 1 & Link/Joint 2 \\
\hline Length & $0.3 \mathrm{~m}$ & $0.3 \mathrm{~m}$ \\
\hline Mass & $1 \mathrm{~kg}$ & $1 \mathrm{~kg}$ \\
\hline Inertia & $0.1 \mathrm{~kg} \mathrm{~m}^{2} / \mathrm{rad}$ & $0.1 \mathrm{~kg} \mathrm{~m}^{2} / \mathrm{rad}$ \\
\hline Viscous friction & $0.1 \mathrm{Nm} \mathrm{s} / \mathrm{rad}$ & $0.1 \mathrm{Nm} \mathrm{s} / \mathrm{rad}$ \\
\hline
\end{tabular}

The state-transformation $x \mapsto \Phi(x)$ and its inverse are

$$
\begin{aligned}
& {\left[\begin{array}{ll}
\xi^{\top} & \eta^{\top}
\end{array}\right]^{\top}=\Phi(x)=\left[\begin{array}{lll}
x_{1}^{\top}-p(\theta)^{\top} & x_{2}^{\top}-\frac{\partial p(\theta)}{\partial \theta}{ }^{\top} \dot{\theta} & z^{\top}
\end{array}\right]^{\top},} \\
& x=\Phi^{-1}(\xi, \eta)=\left[\begin{array}{lll}
\xi_{1}^{\top}+p\left(\eta_{1}\right)^{\top} & \xi_{2}^{\top}+\frac{\partial p\left(\eta_{1}\right)}{\partial \eta_{1}} \eta_{2} & \eta^{\top}
\end{array}\right]^{\top} .
\end{aligned}
$$

For the results presented in this paper, $Q=$ $\operatorname{diag}(10,10,0.1,0.1), R=\operatorname{diag}(0.01,0.01)$ and $P$ according to Assumption 3. The horizon length, sampling time interval and path are $T=0.5 s, \delta=0.01 s$ and $p(\theta)=$ $\left[2 \pi \theta \frac{1}{2} \sin (8 \pi \theta)\right]^{\top}$, respectively. The control bounds on the joint torques are $u \in[-20,20] \times[-10,10]$. The terminal state constraints are defined as $\xi^{\top} P \xi \leq 0.2$ and $\eta \in$ $(-\infty, \infty) \times[-0.05,0.05] \times[-0.05,0.05]$. The economic objective is defined as $F^{\|}=-\eta_{2}+10^{-6} v^{\pitchfork \mathrm{T}} v^{\pitchfork}+10^{-4}\left(v^{\|}\right)^{2}$. The goal is to maximize, in each prediction, the traveled distance $\theta$ along the path, with small regularization terms on the controls.

\section{B. SIMULATION RESULTS}

Fig. 1 shows the traveled distance $\eta_{1}$ by the end of the 5 second simulation for different values of $\epsilon_{\mathcal{N}}$, with the initial state $x_{0}=\mathbb{O}_{6 \times 1}$. As expected, we observe that the traveled distance grows with the size of $\mathcal{N}$; the controller is allowed to cut corners in the benefit of faster motion and successfully utilizes this freedom.

Fig. 2 depicts simulation results for a fixed value of $\epsilon_{\mathcal{N}}=0.3$, with $x_{0}=\left[\begin{array}{lll}0.3 & 0.3 & \mathbb{O}_{1 \times 4}\end{array}\right]^{\top}$, i.e. the robot arm is at a standstill in a perturbed orientation with respect to the fully extended downwards position.. The top plot shows how initially, the state converges to $\mathcal{N}$ from the initial state. The objective of $\mathrm{OCP}^{\|}$is defined such that fast motion is desired. This leads to the actuator bounds being active most of the time and the state $\xi$ remaining close to $\partial \mathcal{N}$ which results in a shorter travel distance. The closed-loop $\xi$-trajectories converge from the initial state to the set $\mathcal{N}$. At $2.5 \mathrm{~s}$ a large disturbance is inflicted, the robot arm is stopped, and both joint angles are changed by $0.6 \mathrm{rad}$. One can observe how the controller successfully stabilizes the set $\mathcal{N}$.

\section{CONCLUSION}

This paper presented a two-stage NMPC control scheme based on transverse feedback linearization. An outer NMPCloop stabilizes a neighborhood of a target manifold. An inner NMPC-loop optimizes tangential performance. The manifold stabilization loop ignores economic performance and generates a convergence bound towards a neighborhood of the manifold. The economic inner NMPC-loop refines the 


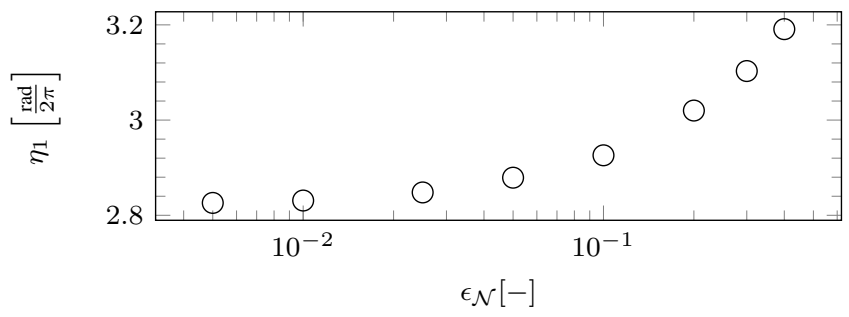

Fig. 1. Distance traveled $\theta=\eta_{1}$ during 5 second simulations for different values of $\epsilon_{N}$, with $x_{0}=\mathbb{D}_{6 \times 1}$.
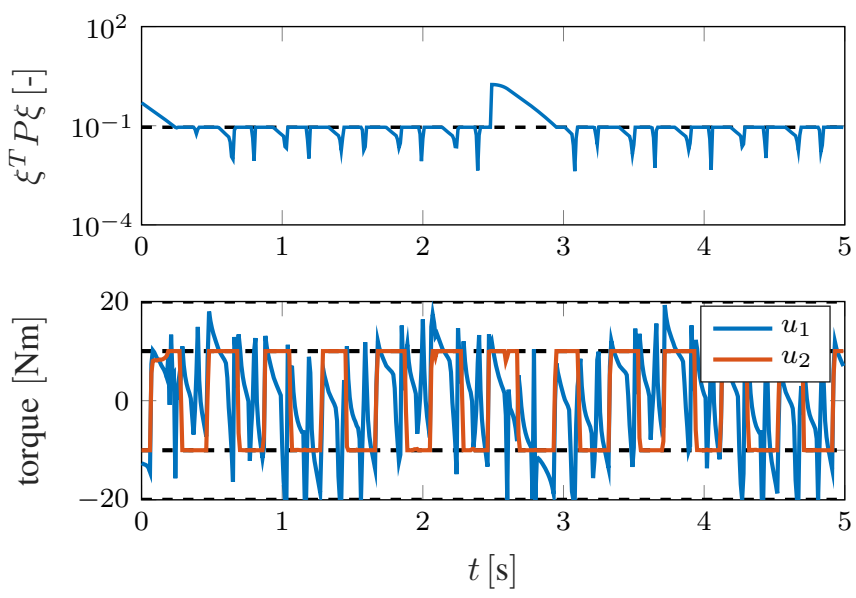

Fig. 2. Closed-loop simulation results. The top figure visualizes the convergence of $\xi$ to $\mathcal{N}$, the dashed line represents $\epsilon_{\mathcal{N}}^{2}$. The bottom figure shows the control input trajectories, bounds are depicted as dashed lines.

trajectories to the benefit of economic performance without compromising convergence. We show that the control scheme leads to convergence to a neighborhood of the target manifold, where a prescribed deviation is allowed for the sake of improving economic performance. Simulation experiments show an example of the application of path-following control applied to a planar robotic manipulator using the proposed control scheme. A time-optimal motion objective on a target manifold is used in the economic tangential NMPC controller. The closed-loop simulation results show that the size of the target neighborhood is highly correlated to the achieved closed-loop economic performance.

\section{REFERENCES}

[1] C. Nielsen and M. Maggiore, "On local transverse feedback linearization," SIAM Journal on Control and Optimization, vol. 47, no. 5, pp. 2227-2250, Jul. 2008.

[2] A. Banaszuk and J. Hauser, "Feedback linearization of transverse dynamics for periodic orbits," Systems \& Control Letters, vol. 26, no. 2, pp. 95-105, 1995.

[3] R. Skjetne, T. I. Fossen, and P. V. Kokotovic, "Robust output maneuvering for a class of nonlinear systems," Automatica, vol. 40, pp. 373-383, 2004.

[4] C. Nielsen and M. Maggiore, "Output stabilization and maneuver regulation: A geometric approach," Systems \& Control Letters, vol. 55, no. 5, pp. 418-427, 2006.

[5] A. P. Aguiar and J. P. Hespanha, "Trajectory-tracking and pathfollowing of underactuated autonomous vehicles with parametric modeling uncertainty," IEEE Transactions on Automatic Control, vol. 52, no. 8, pp. 1362-1379, 2007.
[6] T. Faulwasser, T. Weber, P. Zometa, and R. Findeisen, "Implementation of nonlinear model predictive path-following control for an industrial robot," IEEE Transactions on Control Systems Technology, vol. 25, no. 4, pp. 1505-1511, Sep. 2016.

[7] M. Böck and A. Kugi, "Real-time nonlinear model predictive pathfollowing control of a laboratory tower crane," IEEE Transactions on Control Systems Technology, vol. 22, no. 4, pp. 1461-1473, 2014.

[8] T. Faulwasser and R. Findeisen, "Nonlinear model predictive control for constrained output path following," IEEE Transactions on Automatic Control, vol. 61, no. 4, pp. 1026-1039, 2016.

[9] N. van Duijkeren, R. Verschueren, G. Pipeleers, M. Diehl, and J. Swevers, "Path-following NMPC for serial-link robot manipulators using a path-parametric system reformulation," in Proceedings of the 2016 European Control Conference, Aalborg, Denmark, Jun. 2016, pp. $477-482$.

[10] C. Nielsen, C. Fulford, and M. Maggiore, "Path following using transverse feedback linearization: Application to a maglev positioning system," Automatica, vol. 46, no. 3, pp. 585-590, Mar. 2010.

[11] M. Böck and A. Kugi, "Constrained model predictive manifold stabilization based on transverse normal forms," Automatica, vol. 74, pp. 315-326, Dec. 2016.

[12] J. B. Rawlings, D. Angeli, and C. N. Bates, "Fundamentals of economic model predictive control," in Proceedings of the 51st IEEE Conference on Decision and Control (CDC), Dec. 2012.

[13] M. Ellis, H. Durand, and P. D. Christofides, "A tutorial review of economic model predictive control methods," Journal of Process Control, vol. 24, pp. 1156-1178, 2014.

[14] M. Diehl, R. Amrit, and J. B. Rawlings, "A Lyapunov function for economic optimizing model predictive control," IEEE Transactions on Automatic Control, vol. 56, no. 3, pp. 703-707, 2011.

[15] L. Grüne, "Economic receding horizon control without terminal constraints," Automatica, vol. 49, no. 3, pp. 725-734, Mar. 2013.

[16] T. Faulwasser and D. Bonvin, "On the design of economic NMPC based on approximate turnpike properties," in Proceedings of the 54th IEEE Conference on Decision and Control (CDC), Dec. 2015.

[17] C. Böhm, R. Findeisen, and F. Allgöwer, "Avoidance of poorly observable trajectories: A predictive control perspective," in Proceedings of the 17th IFAC World Congress, 2008, pp. 1952-1957.

[18] J. P. Maree and L. Imsland, "Combined economic and regulatory predictive control," Automatica, vol. 69, pp. 342-347, jul 2016.

[19] L. Grüne and J. Pannek, Nonlinear Model Predictive Control. Springer London, 2011.

[20] A. Isidori, Non-linear control systems. Springer London, 1995.

[21] H. Nijmeijer and A. van der Schaft, Nonlinear Dynamical Control Systems. Springer New York, 1990.

[22] E. Delaleau and J. Rudolph, "Control of flat systems by quasi-static feedback of generalized states," International Journal of Control, vol. 71, no. 5, pp. 745-765, Jan. 1998.

[23] M. Diehl, H. G. Bock, and J. P. Schlöder, "A real-time iteration scheme for nonlinear optimization in optimal feedback control," SIAM Journal on Control and Optimization, vol. 43, no. 5, pp. 1714-1736, 2005.

[24] H. G. Bock, M. Diehl, E. Kostina, and J. P. Schlöder, "Constrained optimal feedback control of systems governed by large differential algebraic equations," in Real-Time PDE-Constrained Optimization. SIAM, 2007, pp. 3-22.

[25] N. van Duijkeren, G. Pipeleers, J. Swevers, and M. Diehl, "Dynamic optimization with partially updated sensitivities," in Proceedings of the 20th IFAC World Congress, Toulouse, France, Jul. 2017.

[26] F. A. Fontes, "A general framework to design stabilizing nonlinear model predictive controllers," Systems \& Control Letters, vol. 42, no. 2, pp. 127-143, 2001.

[27] R. Findeisen, "Nonlinear model predictive control: a sampled-data feedback perspective," Ph.D. dissertation, Universität Stuttgart, 2004.

[28] H. Michalska and R. B. Vinter, "Nonlinear stabilization using discontinuous moving-horizon control," IMA Journal of Mathematical Control and Information, vol. 11, no. 4, pp. 321-340, 1994.

[29] J. Andersson, "A general-purpose software framework for dynamic optimization," PhD Thesis, KU Leuven, 2013.

[30] A. Wächter and L. T. Biegler, "On the implementation of primaldual interior point filter line search algorithm for large-scale nonlinear programming," Mathematical Programming, vol. 106, no. 1, pp. 2557, 2006. 